\title{
Maximum Insertion Rate and Capacity of Multidimensional Constraints
}

\author{
Erez Louidor*, Tze Lei $\mathrm{Poo}^{\dagger}$, Panu Chaichanavong ${ }^{\dagger}$ and Brian H. Marcus* \\ *Department of Mathematics \\ University of British Columbia, Vancouver, BC \\ Email:\{erez, marcus\}@math.ubc.ca \\ ${ }^{\dagger}$ Marvell Semiconductor, Inc. \\ Email:\{lpoo,panuc\}@marvell.com
}

\begin{abstract}
The maximum insertion rate of a one-dimensional constrained system over a finite alphabet is defined to be the maximum density of positions that can be freely, and independently, filled in with arbitrary symbols of the alphabet and still satisfy the constraint. In this paper, this concept is extended to higher dimensional constraints, that is, to constraints on $D$-dimensional arrays defined by imposing a 1-dimensional constraint in each dimension. We give a simple upper bound on the $D$-dimensional maximum insertion rate in terms of the individual 1-dimensional maximum insertion rate. For $D$-dimensional constraints defined by imposing the same 1-dimensional constraint in each dimension, we show that the $D$-dimensional maximum insertion rate is the same as the 1-dimensional maximum insertion rate. In this case (called the isotropic or, sometimes, symmetric case), we show that the maximum insertion rate is a lower bound on the limiting $D$-dimensional capacity as $D$ tends to infinity. Finally, we show that in the case of a finite memory constraint, when the maximum insertion rate is zero, the $D$-dimensional capacity decays exponentially fast to zero.
\end{abstract}

\section{INTRODUCTION}

Typically in digital storage systems source data is encoded twice before written to the medium. First an error-correcting code (or ECC) encodes the source data into a codeword, and then a constrained (or modulation) code is used to transform the codeword into a sequence of bits that can be written reliably to the device. When reading back the data the process is reversed. As the decoder for the constrained code is typically a hard decoder, this scheme has the disadvantage of not making soft information available to the ECC decoder, thereby limiting its error correction capability.

In [8], [6], [7], a reverse concatenation scheme is considered where the user bits are first transformed into a constrained sequence in which certain entries are left unconstrained - i.e., filling them with any combination of bits would result in a sequence satisfying the constraint. Then a systematic ECC of suitable rate is applied, placing the redundancy (parity-check) bits in these unconstrained positions. Since the error correction capability of the ECC depends on the number of redundancy bits, it is desirable that the number of unconstrained positions be as large as possible. On the other hand, increasing the number of unconstrained positions naturally reduces the rate of the constrained encoder. In [7] the authors study the trade- off function that defines for a given "density" of unconstrained positions the maximum rate of the constrained encoder. They also define the maximum insertion rate as essentially the largest asymptotic density of unconstrained positions possible for the constrained code.

In this work, we extend some of these ideas to multidimensional constrained systems. We define the maximum insertion rate of a multidimensional constraint and show that, for isotropic constraints, it is equal to the maximum insertion rate of the underlying 1-dimensional constraint. We also show that the maximum insertion rate of an isotropic constraint is a lower bound on the limiting value of the capacity as the number of dimensions goes to infinity. Finally, we show that for isotropic constraints, whose underlying 1-dimensional constraint has finite memory, when the maximum insertion rate is zero, the capacity decreases to zero exponentially fast as the number of dimensions goes to infinity.

This paper is organized as follows. In Section II we define multidimensional constraints with unconstrained positions, and the maximum insertion rate of such constraints. In Section III we list our results, and in Section IV provide the proofs. Section V presents a conclusion.

\section{Multidimensional CONSTRAints With UNCONSTRAINED POSITIONS}

\section{A. Multidimensional constraints}

Given a finite directed graph whose edges are labeled with symbols from some finite alphabet, each path in the graph corresponds to a finite word attained by reading the labels of the edges of the path in sequence. The path is said to generate the word, and the set of all such generated words is a 1dimensional constrained system or a 1-dimensional constraint. The graph is a presentation of the constraint, and its vertices are usually called states and edges, transitions.

An example of a 1-dimensional constrained system, commonly found in magnetic and optical storage systems, is the $(d, k)$-run length limited constraint, denoted by $\operatorname{RLL}(d, k)$, for nonnegative integers $d \leq k$. This is the set of all binary words that contain at least $d$ zeros between pairs of adjacent ' 1 's and do not contain more than $k$ consecutive ' 0 's anywhere. The $k$ 
parameter is allowed to be $\infty$ which signifies no upper bound on the length of a run of zeros.

Here, we consider multidimensional constraints of dimension $D$, constructed from 1-dimensional constraints, by requiring that all the "rows", in a given direction, of a $D$ dimensional array satisfy a one-dimensional constraint. More precisely, fix a finite alphabet $\Sigma$ and positive integer $D$. For a $D$-tuple $\mathbf{m}=\left(m_{1}, \ldots, m_{D}\right)$ of positive integers, let $\Gamma$ be a $D$-dimensional $m_{1} \times m_{2} \times \ldots \times m_{D}$ array of symbols of $\Sigma$ whose entries are indexed by

$\mathbf{j} \in\left\{0, \ldots, m_{1}-1\right\} \times\left\{0, \ldots, m_{2}-1\right\} \times \ldots \times\left\{0, \ldots, m_{D}-1\right\}$, We will call such an array a $D$-dimensional array of size $\mathbf{m}$. We say that a sequence of $D$-tuples $\left(m_{i}^{(1)}, \ldots, m_{i}^{(D)}\right)_{i=1}^{\infty}$ diverges to infinity if $\left(m_{i}^{(j)}\right)_{i=1}^{\infty}$ diverges to infinity for $j=1,2, \ldots, D$. Given an integer $1 \leq i \leq D$, a row in $d i$ rection $i$ of $\Gamma$ is a sequence of entries of $\Gamma$ of the form $\left(\Gamma_{k_{1}, k_{2}, \ldots, k_{i-1}, j, k_{i+1}, \ldots, k_{D}}\right)_{j=0}^{m_{i}-1}$ for some integers $0 \leq k_{l} \leq m_{l}-1 ; \quad 1 \leq l \leq D, l \neq i$. We say that $\Gamma$ satisfies a $(1-$ dimensional) constraint in direction $i$, if every row in direction $i$ of the array belongs to the constraint. Let $\Sigma^{\mathbf{m}}$ denote all $D$ dimensional arrays of size $\mathbf{m}$ over $\Sigma$, and define $\Sigma^{* * \ldots *}=$ $\Sigma^{*^{D}}$, where the number of $*$ 's in the superscript is $D$, as the union of $\Sigma^{\mathbf{m}}$ over all such $D$-tuples $\mathbf{m}$; that is, $\Sigma^{*^{D}}$ is the set of all finite-size $D$-dimensional arrays. We will deal with subsets of $\Sigma^{*^{D}}$ defined in the following manner. Let $S_{1}, \ldots, S_{D}$ be $D$ (1-dimensional) constraints over $\Sigma$. We denote by $S_{1} \otimes S_{2} \otimes \ldots \otimes S_{D}$ the set of finite $D$-dimensional arrays satisfying the constraint $S_{i}$ in direction $i ; i=1,2, \ldots, D$. We call such a set, a $D$-dimensional constrained system or $D$ dimensional constraint, and by saying that a $D$-dimensional array satisfies the constraint we mean that it belongs to the constraint. Often $S_{1}=S_{2}=\ldots=S_{D}=S$, in which case the constraint $S_{1} \otimes \ldots \otimes S_{D}$ is called isotropic (or symmetric in some papers) and we abbreviate it by $S^{\otimes D}$.

Let $S$ be a $D$-dimensional binary constrained system. For a $D$-tuple $\mathbf{m}=\left(m_{1}, \ldots, m_{D}\right)$, denote by $\mathcal{N}(S, \mathbf{m})$ the number of $D$-dimensional $m_{1} \times m_{2} \times \ldots \times m_{D}$ arrays satisfying $S$, and define $\mathcal{M}(S, \mathbf{m})=(\log \mathcal{N}(S, \mathbf{m})) / \prod_{i=1}^{D} m_{i}$, where the base of the logarithm is 2 and we take $\log (0)$ to be $-\infty$. The capacity of $S$, denoted $\operatorname{cap}(S)$, is defined by

$$
\operatorname{cap}(S)=\lim _{\mathbf{m}_{i} \rightarrow \infty} \mathcal{M}\left(S, \mathbf{m}_{i}\right),
$$

where $\left(\mathbf{m}_{i}\right)_{i=1}^{\infty}$ is a sequence of $D$-tuples diverging to infinity. Since $\log \mathcal{N}(S, \cdot)$ is sub-additive, the above limit exists, is independent of the choice of $\left(\mathbf{m}_{i}\right)_{i=1}^{\infty}$ and satisfies

$$
\operatorname{cap}(S)=\inf _{\mathbf{m}} \mathcal{M}(S, \mathbf{m}) .
$$

See [4] for a proof for $D=2$, which can be generalized for higher dimensions. Assume now that $S$ is a 1-dimensional constraint. Since $\mathcal{M}\left(S^{\otimes(D+1)},\left(m_{1}, \ldots, m_{D}, 1\right)\right)=\mathcal{M}\left(S^{\otimes D},\left(m_{1}, \ldots, m_{D}\right)\right)$

for all positive integers $m_{1}, \ldots, m_{D}$, it follows from (2) that $\operatorname{cap}\left(S^{\otimes(D+1)}\right) \leq \operatorname{cap}\left(S^{\otimes D}\right)$, and hence, $\lim _{D \rightarrow \infty} \operatorname{cap}\left(S^{\otimes D}\right)$ exists. We denote this limit by $\operatorname{cap}_{\infty}(S)$.

\section{B. Unconstrained positions and the maximum insertion rate}

In this section we restrict to the binary alphabet and set $\Sigma=\{0,1\}$. Let $\widehat{\Sigma}$ denote the extended alphabet, $\{0,1, \square\}$, and let $\mathbf{m}$ be a $D$-tuple of positive integers. For a $D$ dimensional array $\Gamma \in \widehat{\Sigma}^{\mathbf{m}}$ with '0's, '1's, and ' $\square$ 's, we define the set of all fillings of $\Gamma$ by, $\Phi(\Gamma)=\left\{\Delta \in \Sigma^{\mathbf{m}}\right.$ : for every index $\mathbf{j}$, if $\Gamma_{\mathbf{j}} \neq \square$ then $\left.\Delta_{\mathbf{j}}=\Gamma_{\mathbf{j}}\right\}$; thus $\Phi(\Gamma)$ contains all the (binary) arrays attained by filling every ' $\square$ ' of $\Gamma$ with ' 0 ' or ' 1 ' independently. Next we define, the set $\widehat{S}$ of $D$-dimensional arrays with entries in $\widehat{\Sigma}$ by,

$$
\widehat{S}=\left\{\Gamma \in \widehat{\Sigma}^{*^{D}}: \Phi(\Gamma) \subseteq S\right\},
$$

thus $\widehat{S}$ contains all the arrays over $\widehat{\Sigma}$ such that every filling of the ' $\square$ 's by '0's and '1's results in an array that satisfies $S$.

If $S$ is a binary 1-dimensional constraint, [7] shows how to construct a presentation for $\widehat{S}$. Thus, $\widehat{S}$ is a 1 -dimensional constrained system. If $S=S_{1} \otimes \ldots \otimes S_{D}$ for some $D$ onedimensional constraints $S_{1}, \ldots, S_{D}$, then it is easily verified that $\widehat{S}=\widehat{S_{1}} \otimes \ldots \otimes \widehat{S_{D}}$, and hence $\widehat{S}$ is a $D$-dimensional constraint as well.

For a binary constrained system $S$, its maximum insertion rate is the maximal asymptotic density of ' $\square$ 's in arrays of $\widehat{S}$ when the array size approaches infinity. More precisely, given an array $\Gamma \in \widehat{\Sigma}^{*^{D}}$, let $\rho(\Gamma)$ denote the density of ' $\square$ 's in $\Gamma$, that is the ratio of the number of ' $\square$ 's to the total number of symbols in $\Gamma$. We define the maximum insertion rate of $S$, denoted $\mu(S)$, by

$$
\mu(S)=\sup _{\left(\Gamma_{i}\right)_{i=1}^{\infty} \subseteq \widehat{S}} \limsup _{i \rightarrow \infty} \rho\left(\Gamma_{i}\right),
$$

where the sup above is taken over all sequences of $D$ dimensional arrays in $\widehat{S}$ whose corresponding sequence of sizes diverges to infinity.

For a binary 1-dimensional constraint, $S$, let $\widehat{G}$ be a presentation for $\widehat{S}$. The density of squares in a path of $\widehat{G}$ is the ratio of the number of edges in the path labeled with a ' $\square$ ', to the total number of edges in the path. In [7] it is shown that $\mu(S)$ is equal to the maximum density of ' $\square$ 's in a simple cycle of $\widehat{G}$, and is therefore rational. Additionally, it is shown that the maximum insertion rate of the RLL constraints is given by

$$
\mu(\operatorname{RLL}(d, k))=\frac{\left\lfloor\frac{k-d}{d+1}\right\rfloor}{\left\lfloor\frac{k+1}{d+1}\right\rfloor(d+1)}
$$

and

$$
\mu(\operatorname{RLL}(d, \infty))=\frac{1}{d+1},
$$

for nonnegative integers $d \leq k$.

\section{MAIN RESULTS}

In this section we list our results. The proofs are provided in the following section.

For multidimensional isotropic constraints constructed from a 1-dimensional constraint $S$, it is somewhat surprising that the maximum insertion rate remains the same. This is stated in the following theorem. 
Theorem 1: Let $S=S_{1} \otimes \ldots \otimes S_{D}$ be a $D$-dimensional constraint, then the following statements hold.

1) $\mu(S) \leq \min \left\{\mu\left(S_{1}\right), \ldots, \mu\left(S_{D}\right)\right\}$.

2) If $S_{1}=\ldots=S_{D}=T$ then $\mu(S)=\mu(T)$.

Since the maximum insertion rate of a 1-dimensional constraint can be computed from a presentation of $\widehat{S}$, it follows from part 2 that the maximum insertion rate of any $D$ dimensional isotropic constraint can also be computed.

The following theorem relates the maximum insertion rate of a constraint with the limiting value of its capacity.

Theorem 2: Let $S$ be a 1-dimensional constraint, then

$$
\operatorname{cap}_{\infty}(S) \geq \mu(S) \text {. }
$$

In [9] the authors show that for $S=\operatorname{RLL}(d, \infty)$, (6) holds with equality. We have been able to show that this is also true for one-dimensional constraints $S$ with maximum insertion rate zero and finite memory. This follows from the next theorem.

A labeled graph $G$ has finite memory $m$, if all paths of length $m$, generating the same word, terminate at the same vertex of $G$. A 1-dimensional constraint has finite memory if it has a presentation with finite memory. The memory of such a constraint is the smallest memory of its finite memory presentations. See [10] for more details. We can now state our next theorem.

Theorem 3: Let $S$ be a 1-dimensional constraint with finite memory $m$. If $\mu(S)=0$ then for all positive integers $D$,

$$
\operatorname{cap}\left(S^{\otimes(D+1)}\right) \leq \frac{m}{m+1} \operatorname{cap}\left(S^{\otimes D}\right) .
$$

In particular $\operatorname{cap}_{\infty}(S)=0$.

As an application of Theorem 3, consider the constraint $\operatorname{RLL}(d, k)$. It has memory $k$, and by equations (4) and (5), $\mu(\operatorname{RLL}(d, k))=0$ if and only if $k \leq 2 d$. Thus by Theorem 3 and Theorem 2, we obtain the following corollary, that was already observed in [5].

Corollary 1: Let $d, k$ be nonnegative integers such that $d \leq$ $k$. Then

$$
\operatorname{cap}_{\infty}(\operatorname{RLL}(d, k))=0 \text { if and only if } k \leq 2 d .
$$

Another family of constraints, found in magnetic and optical recording, is the multiple-spaced runlength constraints denoted $\operatorname{RLL}(d, k, s)$. These are characterized by nonnegative integers $d, k, s$ with $d \leq k$. A binary word satisfies $\operatorname{RLL}(d, k, s)$ if it satisfies $\operatorname{RLL}(d, k)$ and the length of each run of zeros is a multiple of $s$. A storage system employing the $\operatorname{RLL}(2,18,2)$ is described in [2] and [3]. In [1], $\operatorname{RLL}(d, k, 2)$ constraints are studied in the context of magnetic recording.

Now, fix integers $d, k, s$ with $d \leq k$ and $s \geq 2$, and let $S=$ $\operatorname{RLL}(d, k, s)$. It can be verified that each word of $\widehat{S}$ has at most two ' $\square$ 's, and consequently $\mu(S)=0$. As the memory of $S$ is (at most) $k$, by Theorem 3 , we have the following corollary.

Corollary 2: Let $d, k, s$ be nonnegative integers such that $d \leq k$ and $s>1$. Then

$$
\operatorname{cap}\left(\operatorname{RLL}(d, k, s)^{\otimes D}\right) \leq\left(\frac{k}{k+1}\right)^{D-1} \operatorname{cap}(\operatorname{RLL}(d, k, s)) .
$$

\section{PROOFS}

Proof of Theorem 1. We first prove part 1 . Let $1 \leq j \leq D$ be an integer such that $\mu\left(S_{j}\right)=\min \left\{\mu\left(S_{1}\right), \ldots, \mu\left(S_{D}\right)\right\}$. Fix a positive real number $\epsilon$, and let $\left(\Gamma_{i}\right)_{i=1}^{\infty} \subseteq \widehat{S}$ be a sequence of $D$-dimensional arrays with sizes diverging to infinity, such that

$$
\limsup _{i \rightarrow \infty} \rho\left(\Gamma_{i}\right) \geq \mu(S)-\epsilon .
$$

For a positive integer $i$, consider the set of rows in direction $j$ of $\Gamma_{i}$. Since $\rho\left(\Gamma_{i}\right)$ is the average of the densities of ' $\square$ 's in these rows, there exists a row with density of squares at least $\rho\left(\Gamma_{i}\right)$. Let $z_{i}$ be such a row. Clearly $z_{i} \in \widehat{S_{j}}$, and therefore

$$
\mu\left(S_{j}\right) \geq \limsup _{i \rightarrow \infty} \rho\left(z_{i}\right) \geq \limsup _{i \rightarrow \infty} \rho\left(\Gamma_{i}\right) \geq \mu(S)-\epsilon .
$$

Since $\epsilon$ is arbitrary, we have $\mu\left(S_{j}\right) \geq \mu(S)$.

As for part 2 , fix a presentation $\widehat{G}$ for $\widehat{T}$, and let $w=w_{0} \ldots w_{n-1}$ be a word in $\widehat{T}$ generated by a cycle of $\widehat{G}$ with density of ' $\square$ 's equal to $\mu(T)$. For each positive integer $i$, let $\Gamma_{i}$ be the $D$-dimensional array of size $(i n, \ldots, i n)$ with entries given by

$$
\left(\Gamma_{i}\right)_{\left(m_{1}, m_{2}, \ldots, m_{D}\right)}=w_{\left(m_{1}+m_{2}+\ldots+m_{D}\right) \bmod n} .
$$

Then every row (in any direction) of $\Gamma_{i}$ is a cyclic shift of $i$ concatenations of $w$, and is therefore in $\widehat{T}$. Thus, $\left(\Gamma_{i}\right)_{i=1}^{\infty} \subseteq \widehat{S}$ and $\rho\left(\Gamma_{i}\right)=\mu(T)$, for $i=1,2, \ldots$. It follows that $\mu(S) \geq$ $\mu(T)$. On the other hand, by part $1 \mu(S) \leq \mu(T)$, and the result follows.

Proof of Theorem 2. We require the following lemma.

Lemma 1: For any $D$-dimensional constraint $S, \operatorname{cap}(S) \geq$ $\mu(S)$.

Given the lemma, the proof of the theorem is easily completed. Indeed, by the lemma and part 2 of Theorem 1, it follows that $\operatorname{cap}\left(S^{\otimes D}\right) \geq \mu\left(S^{\otimes D}\right)=\mu(S)$ for any positive integer $D$. The theorem follows by taking the limit as $D \rightarrow \infty$.

We now turn to prove the Lemma. Let $\epsilon$ be a positive real number and let $\left(\Gamma_{i}\right)_{i=1}^{\infty} \subseteq \widehat{S}$ be a sequence of $D$ dimensional arrays, with sizes diverging to infinity, satisfying $\limsup _{i \rightarrow \infty} \rho\left(\Gamma_{i}\right) \geq \mu(S)-\epsilon$. Denote by $\mathbf{m}_{i}$ the size of $\Gamma_{i}$, and by $n_{i}$ the product of entries in $\mathbf{m}_{i}$ (i.e., $n_{i}$ is the number of symbols in $\left.\Gamma_{i}\right)$. Then, since each of the $2^{\rho\left(\Gamma_{i}\right) n_{i}}$ ways of replacing the ' $\square$ 's in $\Gamma_{i}$ by ' 0 's and ' 1 's results in a distinct array of $S$, it follows that $\mathcal{M}\left(S, \mathbf{m}_{i}\right) \geq \rho\left(\Gamma_{i}\right)$, and consequently,

$$
\operatorname{cap}(S)=\lim _{i \rightarrow \infty} \mathcal{M}\left(S, \mathbf{m}_{i}\right) \geq \limsup _{i \rightarrow \infty} \rho\left(\Gamma_{i}\right) \geq \mu(S)-\epsilon .
$$

Since $\epsilon$ is arbitrary, the result follows.

Proof of Theorem 3. For simplicity, and in order to highlight the main new concept, we only give the complete proof here for the case when $S$ is irreducible, i.e has an irreducible (strongly connected) presentation. After the proof, we sketch the modifications needed for the reducible case.

We need the following definition. Let $\Sigma=\{0,1\}$. For a word $w \in \Sigma^{*}$ denote by $|w|$ the number of symbols (length) in $w$. We call a 1-dimensional constrained system $S,(m, a)$ determined, for nonnegative integers $m$ and $a$, if there do not 
exist two words $x 0 y, x 1 y \in S$, such that $|x|=m$ and $|y|=a$. Thus, if $w$ is a word of an $(m, a)$-determined constraint, then each symbol placed sufficiently far from the beginning and end of $w$ is uniquely determined by its $m$ preceding symbols and $a$ succeeding symbols.

Now, let $S$ be a general 1-dimensional constraint. If $\mu(S)>0$, then, since there exist arbitrarily long words $x, y \in \Sigma^{*}$ such that $x \square y \in \widehat{S}$, it follows that $S$ is not $(m, a)$-determined for any nonnegative integers $m, a$. It turns out that the converse is also true.

Proposition 1: Let $S$ be an irreducible 1-dimensional constrained system with finite memory $m$. If $\mu(S)=0$ then $S$ is $(m, m)$-determined.

Proof. Let $G$ be an irreducible presentation of $S$ with memory $m$. Assume to the contrary that there exist two words $x 0 y, x 1 y \in S$, where $x, y$ are binary words of length $m$. Let $\quad \alpha_{0} \rightarrow \alpha_{1} \rightarrow \ldots \rightarrow \alpha_{2 m+1}, \quad \beta_{0} \rightarrow \beta_{1} \rightarrow \ldots \rightarrow \beta_{2 m+1}$ be the paths in $G$ generating $x 0 y, x 1 y$ respectively, where $\alpha_{0}, \ldots, \alpha_{2 m+1}, \beta_{0}, \ldots, \beta_{2 m+1}$ are states in $G$. Since $G$ has memory $m$, it follows that $\alpha_{m}=\beta_{m}$ and $\alpha_{2 m+1}=\beta_{2 m+1}$. Since $G$ is irreducible there exists a path $\alpha_{2 m+1} \rightarrow \gamma_{1} \rightarrow \gamma_{2} \rightarrow \ldots \rightarrow \gamma_{l} \rightarrow \alpha_{m}$ connecting $\alpha_{2 m+1}$ to $\alpha_{m}$, and generating some word $z$. Therefore, $G$ contains the cycles $\quad \alpha_{m} \rightarrow \alpha_{m+1} \rightarrow \ldots \rightarrow \alpha_{2 m+1} \rightarrow \gamma_{1} \rightarrow \ldots \rightarrow \gamma_{l} \rightarrow \alpha_{m}$ and $\alpha_{m}=\beta_{m} \rightarrow \beta_{m+1} \rightarrow \ldots \rightarrow \beta_{2 m+1} \rightarrow \gamma_{1} \rightarrow \ldots \rightarrow \gamma_{l} \rightarrow \beta_{m}=\alpha_{m}$, that generate the words $0 y z$ and $1 y z$ respectively. Consequently, for any finite sequence of bits $b_{1}, b_{2}, \ldots, b_{i} \in$ $\Sigma$, the word $b_{1} y z b_{2} y z \ldots b_{i} y z \in S$. Hence, $(\square y z)^{i} \in \widehat{S}$, for all positive integers $i$, contradicting our assumption that $\mu(S)=0$.

The following proposition shows that if $S$ is an $(m, a)$ determined constraint, the capacity of $S^{\otimes D}$ decreases at least exponentially fast with $D$. This is a generalization of [5, Lemma 3] where $S=\operatorname{RLL}(d, k)$ with $k \leq 2 d$. In fact the proof given there works for general $(m, a)$-determined constraints as well, and we reproduce it here in our setting.

Proposition 2: Let $S$ be a 1-dimensional $(m, a)$-determined constraint. Then for all positive integers $D$

$$
\operatorname{cap}\left(S^{\otimes(D+1)}\right) \leq \frac{b}{b+1} \operatorname{cap}\left(S^{\otimes D}\right),
$$

where $b=\min \{m, a\}$.

Proof. We will prove this for the case $b=a$. The case $b=m$ is handled similarly. Let $l$ be a positive integer. Denote by $l$ the $D$-tuple with every entry equal to $l$, and let $\mathbf{m}_{l}$ be the $(D+1)$-tuple given by $\mathbf{m}_{l}=(m+l(a+1), l, l, \ldots, l)$. Recall that $\mathcal{N}\left(S^{\otimes(D+1)}, \mathbf{m}_{l}\right)$ is the number of arrays in $S^{\otimes(D+1)}$ of size $\mathbf{m}_{l}$. We will bound $\mathcal{N}\left(S^{\otimes(D+1)}, \mathbf{m}_{l}\right)$ from above. For an array $\Gamma$ of size $\mathbf{m}_{l}$ and integer $0 \leq i<m+l(a+1)$, we denote by $\Gamma^{(i)}$ the $D$-dimensional sub-array of $\Gamma$ of size 1 , consisting of the entries $\Gamma_{i, \mathbf{j}} ; \mathbf{j} \in\{0,1, \ldots, l-1\}^{D}$. Next, let $i_{r}=m+r(a+1) ; r=0,1, \ldots, l-1$, and set

$$
A=\{0,1, \ldots, m+l(a+1)-1\} \backslash\left\{i_{r}: r=0, \ldots, l-1\right\} .
$$

Clearly for each array $\Gamma$ of size $\mathbf{m}_{l}$ in $S^{\otimes(D+1)}$, each of the sub-arrays $\Gamma^{(i)}$ satisfies $S^{\otimes D}$. We will construct all such arrays
$\Gamma$ by first selecting the sub-arrays $\Gamma^{(k)} ; k \in A$ - each chosen from the set of arrays of size 1 in $S^{\otimes D}-$ and then completing the entries of the sub-arrays $\Gamma^{\left(i_{r}\right)} ; r=0,1, \ldots, l-1$. We will show that for each possible choice of the sub-arrays $\Gamma^{(k)} ; k \in A$ there is at most one possibility to complete the sub-arrays $\Gamma^{\left(i_{r}\right)} ; r=0,1, \ldots, l-1$ such that the resulting array $\Gamma$ satisfies $S^{\otimes(D+1)}$. For $\mathbf{j} \in\{0,1, \ldots, l-1\}^{D}$, consider the sequence of entries $\Gamma_{\mathbf{j}}^{(0)}, \Gamma_{\mathbf{j}}^{(1)}, \ldots, \Gamma_{\mathbf{j}}^{(m+l(a+1)-1)}$. It is a row in direction 1 of $\Gamma$, and contains the yet unselected entries: $\Gamma_{\mathbf{j}}^{\left(i_{0}\right)}, \ldots, \Gamma_{\mathbf{j}}^{\left(i_{l-1}\right)}$. We complete these entries in sequence. The entry $\Gamma_{\mathbf{j}}^{\left(i_{0}\right)}$ is preceded by $m$, already selected, entries in the row and succeeded by $a$, already selected, entries in the row. Thus, since $S$ is $(m, a)$-determined, there is at most one possibility to complete it. For $r \geq 1$, after completing the entries $\Gamma_{\mathbf{j}}^{\left(i_{k}\right)}$, for $0 \leq k<r$ the entry $\Gamma_{\mathbf{j}}^{\left(i_{r}\right)}$ is preceded by (at least) $m$ completed entries in the row, and succeeded by $a$ completed entries in the row. Hence, again, there is at most one possibility to complete it. Therefore, indeed, for each selection of the sub-arrays $\Gamma^{(k)} ; k \in A$ there is at most one possibility to complete the rest of $\Gamma$ so that the resulting array satisfies $S^{\otimes(D+1)}$. Since there are $\mathcal{N}\left(S^{\otimes D}, \mathbf{l}\right)^{m+l a}$ ways to select the sub-arrays $\Gamma^{(k)} ; k \in A$, it follows that $\mathcal{N}\left(S^{\otimes(D+1)}, \mathbf{m}_{l}\right) \leq \mathcal{N}\left(S^{\otimes D}, \mathbf{l}\right)^{m+l a}$. Hence,

$$
\begin{aligned}
\mathcal{M}\left(S^{\otimes(D+1)}, \mathbf{m}_{l}\right) & =\frac{\log \mathcal{N}\left(S^{\otimes(D+1)}, \mathbf{m}_{l}\right)}{l^{D}(m+l(a+1))} \\
& \leq \frac{(m+l a) \log \mathcal{N}\left(S^{\otimes D}, \mathbf{l}\right)}{l^{D}(m+l(a+1))} \\
& =\frac{m+l a}{m+l(a+1)} \mathcal{M}\left(S^{\otimes D}, \mathbf{l}\right)
\end{aligned}
$$

Taking the limit as $l \rightarrow \infty$, we obtain the desired result. ${ }^{1}$

The proof of Theorem 3 is now easily completed. Let $S$ be an irreducible 1-dimensional constraint with finite memory $m$. By Proposition $1, S$ is $(m, m)$-determined, and the theorem follows from Proposition 2.

A reducible constraint with finite memory and $\mu(S)=0$ need not be $(m . a)$-determined for any $m$ and $a$. For example, consider the constraint $S$ consisting of all sequences of the form $0^{u} 1^{v}$ where $u, v$ are arbitrary nonnegative integers. This constraint is presented by a graph with two states $a, b$, a selfloop labeled 0 at $a$, an edge from $a$ to $b$ labeled 1 , and a selfloop at $b$ labeled 1 . This presentation has finite memory, but $S$ is reducible since a 0 can never follow a 1 . And $\mu(S)=0$ since any string in $\hat{S}$ can contain at most one $\square$ (at a transition from 0 to 1$)$. But $S$ is not $(m, a)$ determined for any $m, a$ since $0^{m} x 1^{a}$ can be filled in by $x=0$ or $x=1$. Of course, there can be much more complicated constraints with these features.

Now, let $S$ be any 1-dimensional constraint with finite memory $m$ and $\mu(S)=0$. For each sequence $x$ in $S$ and positive integer $N$, let $V_{N}(x)$ be the set of sequences $y$ in $S$ such that $y_{i}=x_{i}$ whenever $i$ is not divisible by $N$. By using

\footnotetext{
${ }^{1}$ Observe that by fixing the size of the first dimension, we can also obtain a bound on the capacity of the $D$-dimensional "strip" constraint, which is an upper bound on the capacity of the $D+1$ dimensional constraint. However the resulting bound is weaker.
} 
the general structure of reducible graphs [10](section 2.5.1), one can show that $\left|V_{m+1}(x)\right|$ is uniformly bounded over all $x$ in $S$. Armed with this property, the proof of Proposition 2 can be modified to give the same result with $b=m$, and this gives the proof of Theorem 3 in the reducible case.

\section{CONCLUSION}

The maximum insertion rate of a constraint is a trivial lower bound on its capacity. We showed a simple upper bound on the maximum insertion rate for a constrained system. For isotropic $D$-dimensional constraints, it turns out that the maximum insertion rate is equal to the maximum insertion rate of the underlying 1-dimensional constraint. For such constraints, the maximum insertion rate provides a lower bound on the limiting value of the capacity as the number of dimensions grows to infinity. We showed that for isotropic constraints with maximum insertion rate 0 , in which the underlying 1 dimensional constraint has finite memory, the limiting value of the capacity is in fact 0 and the rate of convergence is exponential.

\section{REFERENCES}

[1] P. FUNK, "Run-length-limited codes with multiple spacing, " IEEE Trans. Magnetics, 18 (1982), 772-775.
[2] R. Haeb, D. Rugar, T. Howell and P.Coleman, "Coding and signal processing for a magnetooptic resonant bias coil overwrite experiment," IBM Research Report, RJ 6962 (66499), 1989.

[3] D. RugAR, P.H. SiEGEL, "Recording results and coding considerations for the resonant bias coil overwrite technique," Optical Data Storage Topical Meeting, G.R. Knight, C.N. Kurtz (Editors), Proc. SPIE, Vol. 1078 (1989), 265-270.

[4] A. KATO AND K. ZEger, "On the capacity of two-dimensional run length constrained channels," IEEE Trans. Inform. Theory, 45 (1999), 1527-1540.

[5] H. Ito, A. Kato, Zs. NAGY, AND K. Zeger, "Zero capacity region of multidimensional run length constraints," The Electronic Journal of Combinatorics vol. 6(1), no. R33, 1999.

[6] J. C. De Souza, B. H. Marcus, R. New, and B. A. Wilson, Constrained systems with unconstrained positions, IEEE Trans. Inform. Theory, 48 (2002), 866-879.

[7] T. L. Poo, P. Chaichanavong, and B. H. Marcus, "Trade-off functions for constrained systems with unconstrained positions," IEEE Trans. Inform. Theory, 52 (2006), 1425-1449.

[8] A. J. VAN WiJngaARden AND K. A. S. ImMinK, "Maximum runlength-limited codes with error control capabilities," IEEE J. Select. Areas Commun., 19 (2001), 602-611.

[9] E. ORDENTLICH AND R.M. ROTH, "Independent sets in regular hypergraphs and multi-dimensional runlength-limited constraints," SIAM J. Disc. Math., 17 (2004), 615-623.

[10] B. H. Marcus, R. M. Roth, and P. H. Siegel, "An Introduction to Coding for Constrained Systems," Lecture notes www.cs.technion.ac.il/ ronny/constrained.html

[11] R. M. Roth, P. H. Siegel, AND J. K. Wolf, "Efficient coding schemes for the hard-square model," IEEE Trans. Inform. Theory, 47 (2001), $1166-1176$. 